\title{
VERIFICATION AND TESTING OF CONCURRENT SYSTEMS WITH ACTION RACES
}

\author{
Alex Petrenko and Andreas Ulrich ${ }^{\mathrm{b}}$ \\ a Centre de Recherche Informatique de Montreal, 550 Sherbrooke West, Suite 100, Montreal, \\ H3A $1 B 9$, Canada \\ E-mail: petrenko@crim.ca \\ b Siemens AG, Corporate Technology, ZT SE 1, 81730 Munich, Germany \\ E-mail: andreas.ulrich@mchp.siemens.de
}

\begin{abstract}
We propose a categorization of action race conditions which occur in the specification of a concurrent system given as a collection of communicating labeled transition systems. We present several conditions to detect action races in the concurrent system operating in three different environments: a sequential-slow environment that allows execution of external actions only in stable states, a sequential-fast environment that supports the sequential execution of an action sequence before the next stable state is reached, and a concurrent environment that performs independent actions simultaneously. Race analysis facilitates the design of testers that can execute test actions without races at a highest possible speed.
\end{abstract}

Keywords: Concurrent systems, communicating LTS, action race analysis, race-free testing, test execution.

\section{INTRODUCTION}

It is understood that races, besides interleaving of concurrent actions and internal nondeterminism, are a form of nondeterminism that is often inherent to concurrent systems. Depending on the execution order of actions, the global state of the system reached after a race is ambiguous.

In general, races are a source of software faults that are difficult to detect during test execution. They require repeated test runs of the same test case with varying execution speeds of test events and an analysis of the test result

The original version of this chapter was revised: The copyright line was incorrect. This has been corrected. The Erratum to this chapter is available at DOI: 10.1007/978-0-387-35516-0_20 
after each test run. As an alternative to testing, possible races can be analyzed based on a formal specification of the concurrent system within the design phase. However, general verification methods do not pay particular attention to race conditions and other types of nondeterminism since they evaluate all possible execution sequences of the system in a systematic manner [5]. Therefore, analysis techniques to detect race conditions are needed.

Work on race analysis started first in the context of sequential circuits [3]. Here, a distinction between race and race condition was introduced: a race condition results into a race if a circuit reaches different destination states during execution. The existence of races is explained from the specification of the circuits only.

Race analysis of concurrent software systems has mostly concentrated on an analysis of traces observed during the execution of the concurrent system. The approach in [11] assumes no specification of the concurrent system. Instead it tries to deduce possible race conditions from the mere observation of traces. The test engineer has to decide whether a certain constellation of message exchanges really constitutes a race. The trace analysis is based on a suitable definition of a happen-before relation between messages [4]. Similar work is presented in [9]. Another approach tries to detect races on-the-fly during the execution of a concurrent program using probing software [2]. Its drawback however is that races remain undiscovered if the suitable trace is not executed. A more systematic approach to trace analysis is presented in [1]. It discusses possible races based on an analysis of the system's behavior using different communication schemas like asynchronous communication with or without message queues.

In this paper we use another approach to detect races. We provide a characterization of races based on available formal specifications of the components of a concurrent system. We show that races occur due to conflicts between internal actions used for internal communication between the components and external actions that the system uses to communicate with its environment. Depending on the speed at which the environment can execute actions with the system, different race conditions occur. To distinguish this type of races from message or data races that are caused due to the underlying communication infrastructure, we call them action races.

The paper is organized as follows. Section 2 introduces basic notations. Section 3 presents definitions of races in the context of different environments. Section 4 applies the findings to construct test sequences that guarantee a race-free test execution. Section 5 concludes the paper. 


\section{ASSUMPTIONS AND DEFINITIONS}

A concurrent system comprises a number of independent components communicating with each other via message passing. Inputs and outputs of a component are not distinguished. Furthermore, there are no spontaneous transitions within a component. Each component has ports to communicate synchronously with the environment or with other components. The concurrent system is open, i.e., it is reactive with its environment.

More formally, a concurrent system $\mathfrak{I}$ is modeled by a system of $n$ finite labeled transition systems (LTSs) communicating synchronously (rendezvous communication). Both, two-way rendezvous and multi-rendezvous, are considered. Each LTS defines a component of the concurrent system. Transmitting messages and their receipt through ports are referred to actions in an LTS.

Definition 1. A labeled transition system (LTS) $M$ is defined by a quadruple $\left(S, A \cup\{\tau\}, \rightarrow, s_{0}\right)$, where $S$ is a finite set of states; $A$ is a finite set of actions; $\rightarrow \subseteq S \times(A \cup\{\tau\}) \times S$ is a transition relation; and $s_{0} \in S$ is the initial state. Symbol $\tau$ denotes an unobservable action of $M$.

A transition $\left(s_{1}, a, s_{2}\right) \in \rightarrow$ is also written as $s_{1}-a \rightarrow s_{2}$. Let $s=a \Rightarrow s^{\prime}$ denote the sequence $s-\tau \rightarrow \ldots-a \rightarrow \ldots-\tau \rightarrow s^{\prime}$. Furthermore, let $s=\sigma \Rightarrow s^{\prime}$ express the sequence $s=a_{1} \Rightarrow s_{1}=a_{2} \Rightarrow s_{2}=\ldots \Rightarrow s^{\prime}$, where $\sigma=a_{1} a_{2} \ldots$ We assume that each component LTS is initially connected, i.e. for each state $s^{\prime}$ there exists a trace $\sigma$ such that $s_{0}=\sigma \Rightarrow s^{\prime}$. Since an LTS is in general nondeterministic, the state $s^{\prime}$ reached after execution of trace $\sigma$ might be not unique. Let therefore ( $s$ after $\sigma)$ denote the set $\left\{s^{\prime} \mid s=\sigma \Rightarrow s^{\prime}\right\}$.

Definition 2. An LTS $M$ is deterministic if $\mid($ s after $\sigma) \mid \leq 1$ for all $\sigma \in A^{*}$ and $s \in S$.

We further consider concurrent systems composed of deterministic LTSs without the unobservable action $\tau$ (rigid LTSs). Their behavior is characterized in terms of traces. The set of traces of state $s$ is defined as the set $\{\sigma \in$ $\left.A^{*} \mid \exists s^{\prime} \in S: s=\sigma \Rightarrow s^{\prime}\right\}=\operatorname{Tr}(s)$. The set of traces of the LTS $M$ is $\operatorname{Tr}\left(s_{0}\right)$.

The composition of two deterministic LTSs $P$ and $Q$ defined over the action sets $A_{P}$ and $A_{Q}$, respectively, to a composite machine is expressed using the composition operator $\|: \operatorname{LTS}\left(A_{P}\right) \times \operatorname{LTS}\left(A_{Q}\right) \rightarrow \operatorname{LTS}\left(A_{P} \cup A_{Q}\right)$, where $\operatorname{LTS}(A)$ denotes the set of all possible LTSs over action set $A$. 
Definition 3. Given two deterministic LTSs $P=\left(S_{P}, A_{P}, \rightarrow_{P}, s_{P}\right)$ and $Q=$ $\left(S_{Q}, A_{Q}, \rightarrow_{Q}, s_{Q}\right)$, the parallel composition operator $\|$ is defined by the following inference rules:

1. If $P-a \rightarrow P^{\prime}, a \notin A_{Q}$ then $(P \| Q)-a \rightarrow\left(P^{\prime} \| Q\right)$.

2. If $Q-a \rightarrow Q^{\prime}, a \notin A_{P}$ then $(P \| Q)-a \rightarrow\left(P \| Q^{\prime}\right)$.

3. If $P-a \rightarrow P^{\prime}, Q-a \rightarrow Q^{\prime}$ then $(P \| Q)-a \rightarrow\left(P^{\prime} \| Q^{\prime}\right)$.

Applied to a given concurrent system, this operator defines a composite machine. States of this machine are called global opposed to local states of component LTSs.

Definition 4. A composite machine $C_{\mathfrak{I}}=M_{1}\|\ldots\| M_{n}$ of a given concurrent system $\mathfrak{I}$ of $n$ LTSs $M_{i}=\left(S_{i}, A_{i}, \rightarrow_{i}, s_{0 i}\right)$ is the LTS $\left(S_{\mathfrak{I}}, A_{\mathfrak{I}}, \rightarrow_{\mathfrak{I}}, s_{0 \mathfrak{I}}\right)$, where $s_{0 \Im}=\left(s_{01}, \ldots, s_{0 n}\right)$ is the initial global state; $S_{\mathfrak{I}} \subseteq S_{1} \times \ldots \times S_{n}, A_{\mathfrak{I}} \subseteq A_{1} \cup \ldots$ $\cup A_{n}$, and $\rightarrow_{\mathfrak{I}} \subseteq S_{\mathfrak{I}} \times A_{\mathfrak{I}} \times S_{\mathfrak{I}}$ are the smallest sets obtained by application of the parallel composition operator II.

For simplicity, we assume that $A_{\mathfrak{I}}=A_{1} \cup \ldots \cup A_{n}$. Let $N$ be the set of indices $\{1, \ldots, n\}$ of the LTSs in $\mathfrak{I}$. Given action $a \in A_{\mathfrak{g}}$, id $(a)$ denotes the occurrence set $\left\{i \in N \mid a \in A_{i}\right\}$ of $a$. We assume that in a given system $\mathfrak{I}$ there are no isolated components, i.e., each component has at least an action $a$ such that $|i d(a)|>1$. Given a subset of actions $A_{\text {ext }} \subseteq A_{\mathfrak{J}}$, actions in $A_{\text {ext }}$ are called external actions, if $A_{\text {ext }} \supseteq\left\{a \in A_{\mathfrak{S}}|| \boldsymbol{i d}(a) \mid=1\right\}$. We do not consider here autonomous systems, which have the empty set of external actions $A_{\text {ext }}$ and we always assume $A_{\text {ext }} \neq \varnothing$. So, a concurrent system as defined here is an open system: It needs an environment to execute external actions. The set of actions of $\mathfrak{I}$ that do not belong to $A_{\text {ext }}$ is the set of internal actions $A_{\text {int }}=A_{\mathfrak{I}} \backslash$ $A_{\text {ext }}$ of system $\mathfrak{I}$. We shall refer to a (local or global) transition as internal or external if it is defined by an internal or external action, respectively.

Let enabled $(s)=\left\{a \mid \exists s^{\prime} \in S_{\mathfrak{I}}:\left(s, a, s^{\prime}\right) \in \rightarrow_{\mathfrak{I}}\right\}$ denote the set of actions that belong to global transitions enabled in global state $s$. A stable global state is a state $s$ such that enabled $(s) \subseteq A_{\text {ext }}$; a transient global state is a state $s$ such that enabled $(s) \neq \varnothing$ and enabled $(s) \not \subset A_{\text {ext }}$. A deadlock state $s$ is defined as a global state with no enabled actions, $\operatorname{enabled}(s)=\varnothing$. We assume in this paper that a concurrent system has at least one stable global state (the initial one) in order to exclude "oscillating" systems.

\section{EXAMPLE}

Figure 1 shows the topology of an example system $\mathfrak{I}$ comprising three components communicating via the internal actions $x, y$, and $z$. The whole system communicates via the external actions $a$ and $b$ with environment $E$. Figure 2 
depicts the behavior of each component in $\mathfrak{I}$ as a LTS. The composite machine defining the global behavior of the system $\mathfrak{I}$ is shown in Figure 3 . In this figure, the bold circles refer to stable states.

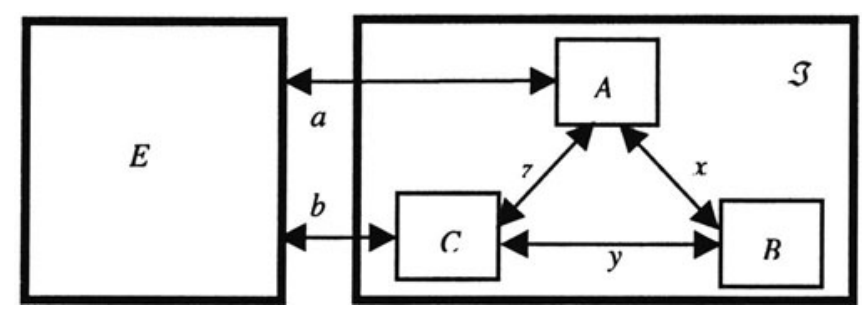

Figure 1. Example system $\mathfrak{I}=\{A, B, C\}$ and environment $E$.
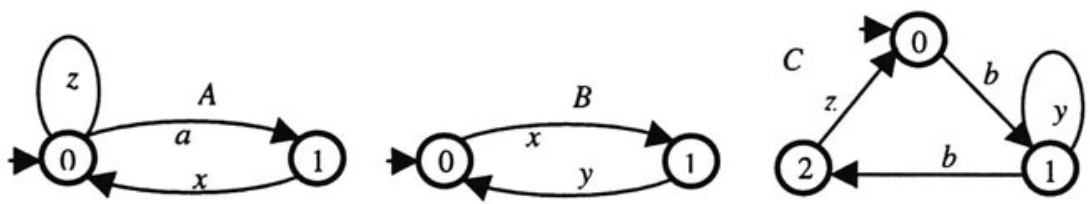

Figure 2. Component LTSs $A, B$, and $C$.

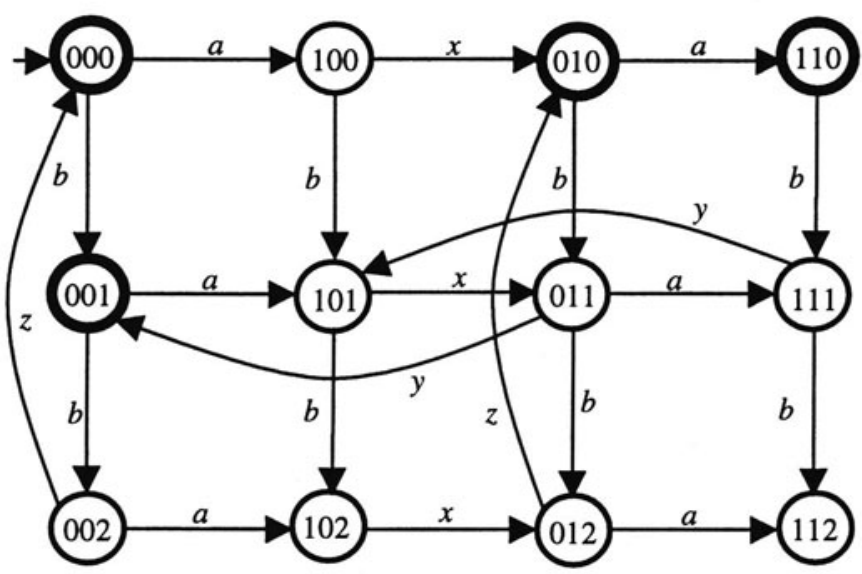

Figure 3. The composite machine $C_{\mathfrak{I}}$ of concurrent system $\mathfrak{I}$. 


\section{RACES IN CONCURRENT SPECIFICATIONS}

\subsection{Race Conditions Categorization}

Intuitively in our model of synchronous communication, an action race condition arises when in a given global state several actions are enabled. A race condition leads to a race when, as a result, the system may reach different destination (stable) states. Note that this phenomenon is pretty much the same as (critical) races in sequential circuits [3].

Depending on types of actions creating race conditions, we distinguish three types of race conditions:

- internal actions race conditions or $i$-race conditions;

- external actions race conditions or e-race conditions;

- mixed (internal and external) race conditions or $m$-race conditions.

Autonomous (closed) systems may have only i-race conditions. A given (open) concurrent system may or may not have a particular type of race conditions if it is put into different environments. Accordingly, we define three basic types of environments depending on its ability to offer either single or several concurrent actions at a time when the system is in a stable or in any (stable or transient) state.

- An environment that submits a single external action at a time only when the concurrent system is in a stable state is called a sequential-slow environment. Here only i-race conditions can occur.

- An environment that may submit external actions sequentially even before the concurrent system has reached the next stable state is called a sequential-fast environment. M-race conditions (in addition to i-race conditions) occur in a system within such an environment.

- Finally, if the environment can offer simultaneously several external actions starting from a stable state, it is called a concurrent environment. Note that since these external actions can be executed in any possible interleaving, a concurrent environment is also a sequential-fast environment. E-race conditions may arise within such an environment.

In this section, we formally define races that can occur in a given concurrent system placed within various environments and discuss the ways to detect them.

\subsection{Sequential-slow Environment}

A sequential-slow environment waits until a concurrent system completes the execution of internal actions before it offers a next external action. The behavior of a concurrent system that is controlled by a sequential-slow envi- 
ronment can be represented by a proper submachine of a composite machine. To define such a machine, we first introduce a modified composition operator for two LTSs, $P$ and $Q$, with a fixed set of external actions $A_{\text {ext }} \subseteq A_{P} \cup$ $A_{Q}$, as the mapping $\rfloor A_{\text {ext }} L: \operatorname{LTS}\left(A_{P}\right) \times \operatorname{LTS}\left(A_{Q}\right) \rightarrow \operatorname{LTS}\left(A_{P} \cup A_{Q}\right)$.

Compared to the usual parallel composition operator II, the new operator prohibits external actions from execution whenever the system can execute an internal action.

Definition 5. Given two LTSs $P=\left(S_{P}, A_{P}, \rightarrow_{P}, s_{P}\right)$ and $Q=\left(S_{Q}, A_{Q}, \rightarrow_{Q}, s_{Q}\right)$ and the sets $A_{\text {ext }}$ and $A_{\text {int }}$ of external and internal actions, respectively, $A_{\text {ext }} \cup$ $A_{\text {int }}=A_{P} \cup A_{Q}$, the $A_{\text {ext }}$-slow composition operator $\rfloor A_{\text {ext }}$ is defined by the following inference rule:

If there exists $a \in A_{\text {int }}$ such that $P-a \rightarrow P^{\prime}, Q-a \rightarrow Q^{\prime}$ then $\left.\left.(P\rfloor A_{\text {ext }} L Q\right)-a \rightarrow\left(P^{\prime}\right\rfloor A_{\text {ext }} L Q^{\prime}\right)$ otherwise 1. If $P-a \rightarrow P^{\prime}, a \notin A_{Q}$ then $\left.(P\rfloor A_{\text {ext }} L Q\right)-a \rightarrow\left(P^{\prime}\right\rfloor A_{\text {ext }}\lfloor Q)$.

2. If $Q-a \rightarrow Q^{\prime}, a \notin A_{P}$ then $(P\rfloor A_{\text {ext }}\lfloor Q)-a \rightarrow(P\rfloor A_{\text {ext }}\left\lfloor Q^{\prime}\right)$.

3. If $P-a \rightarrow P^{\prime}, Q-a \rightarrow Q^{\prime}$ then $\left.\left.(P\rfloor A_{\text {ext }} L Q\right)-a \rightarrow\left(P^{\prime}\right\rfloor A_{\text {ext }} L Q^{\prime}\right)$.

Applied to a given concurrent system, this operator defines the following composition.

Definition 6. Given the concurrent system $\mathfrak{I}$ of $n$ LTSs $M_{i}=\left(S_{i}, A_{i}, \rightarrow_{i}, s_{0 i}\right)$ with the set of external actions $A_{\text {ext }}$, a slow-composite machine $S c_{\mathfrak{I}}=$ $\left.\left.M_{1}\right\rfloor A_{\text {ext }} L \ldots\right\rfloor A_{\text {ext }} L M_{n}$ of $\mathfrak{I}$ is the LTS $\left(S_{S c}, A_{S c}, \rightarrow_{S c}, s_{0 S c}\right)$, where $s_{0 S_{c}}=s_{0 \Im}$ is the initial stable state; $S_{S c} \subseteq S_{1} \times \ldots \times S_{n}, A_{S c} \subseteq A_{1} \cup \ldots \cup A_{n}$, and $\rightarrow_{s c} \subseteq$ $S_{\mathfrak{I}} \times A_{\mathfrak{I}} \times S_{\mathfrak{I}}$ are the smallest sets obtained by application of the $A_{\text {ext }}$-slow composition operator $\rfloor A_{\text {ext }} L$.

For simplicity, we assume that $A_{\text {ext }} \subseteq A_{S c}$. When no confusion arises, we omit the set $A_{\text {ext }}$ from the operator to use $\rfloor$ L. The relationships between the two types of composite machines are stated as follows.

Proposition 1. Given a concurrent system $\mathfrak{I}$ and its composite machine $C_{\mathfrak{I}}$, the slow-composite machine $S c_{\mathfrak{I}}$ is a submachine of $C_{\mathfrak{J}}$ for any set of external actions $A_{\text {ext }}$. If $A_{\text {ext }}=A_{\Im}$, i.e., if the system has no internal actions, then the machines $S c_{\mathfrak{I}}$ and $C_{\mathfrak{I}}$ coincide.

Let ( $s$ after-ext $a$ ) denote the set of all possible stable global states that can be reached by $\mathfrak{I}$ when external action $a$ is executed in stable global state $s$, i.e., $(s$ after-ext $a)=\left\{s^{\prime} \mid \exists \sigma \in A_{\text {int }}^{*}: s^{\prime} \in(s\right.$ after $\left.a \sigma) \wedge \operatorname{enabled}\left(s^{\prime}\right) \subseteq A_{\text {ext }}\right\}$. 
Definition 7. Given stable global state $s$ and external action $a \in$ enabled $(s)$, - system $\mathfrak{I}$ is divergent, i.e., it has a livelock for $a$ in $s$ if ( $s$ after-ext $a)=\varnothing$;

- the action $a$ creates a race in $s$ if $\mid(s$ after-ext $a) \mid>1$;

- the action $a$ is race-free in $s$ if $\mid(s$ after-ext $a) \mid=1$.

The system $\mathfrak{I}$ is said to be race-free within a sequential-slow environment if in each stable state $s$ every $a \in \operatorname{enabled}(s)$ is race-free.

In the sequel we consider only convergent concurrent systems, i.e. systems without livelock. All races in such systems within a sequential-slow environment can be characterized in a so-called stable composite machine defined to be as follows.

Definition 8. Given a concurrent system $\mathfrak{I}$ with the slow-composite machine $S c_{\mathfrak{S}}$, a stable composite machine is an LTS $\left(S_{S t}, A_{S t}, \rightarrow_{S t}, s_{0 S t}\right)$, denoted $S t_{\Im}$, where $s_{0 S t}=s_{0 S c} ; S_{S t} \subseteq S_{S c}, A_{S t} \subseteq A_{\text {ext }}$, and $\rightarrow_{S t} \subseteq S_{S t} \times A_{\text {ext }} \times S_{S t}$ are the smallest sets obtained by application of the following rule: $\left(s, a, s^{\prime}\right) \in \rightarrow_{s t}$ iff $s^{\prime} \in(s$ after-ext $a)$.

For simplicity, we assume that $A_{S t}=A_{\text {ext }}$. A stable composite machine $S t_{\Im}$ can be obtained from the slow composite machine by replacing all internal actions with the unobservable action $\tau$ and subsequently hiding $\tau$ in the obtained LTS by performing the failure-equivalent transformation as defined in [8]. Then, a nondeterministic stable composite machine indicates the presence of races.

Proposition 2. Concurrent system $\mathfrak{I}$ is race-free within a sequential-slow environment iff its stable composite machine $S t_{\mathfrak{S}}$ is deterministic.

Any action sequence of a deterministic stable composite machine is race-free when it is executed within a sequential-slow environment.



Figure 4. Stable composite machine $S t_{\mathfrak{I}}$ of system $\mathfrak{I}$. 


\section{EXAMPLE}

Figure 4 shows the stable composite machine for our working example that is deterministic. This means that the given concurrent system is race-free within a sequential-slow environment. Moreover, as one can see from the composite machine (Figure 3), at most one internal action is enabled in each global state. I-race conditions do not occur in such a system.

The number of states of a stable composite machine may reach that of a composite machine in the worst case situation. The question arises whether races can be detected without actually constructing the full (stable) composite machine. To check i-race conditions there is no need to store the whole stable composite machine, as each combination of external action and stable state can be checked separately.

Some sufficient conditions for the absence of i-race conditions can be established based on the following observation. Internal actions simultaneously enabled in a global state do not create a race if they belong to different local states, as the final state does not depend on the order of their execution. Only internal actions enabled at the same local state may create a race, thus we have the following sufficient condition.

Proposition 3. Concurrent system $\mathfrak{I}$ is race-free within a sequential-slow environment if there exists at most a single internal transition from every local state.

As follows from the definition, this type of action races is completely out of control of the environment in spite of its slow sequential behavior. If a given specification has races within a sequential-slow environment then we may conclude that either there exists a design error (in other words, it has unintended nondeterminism [11]) or the specification is, in fact, an abstraction of a future design and has to be refined further.

\subsection{Sequential-fast Environment}

Assume now that a concurrent system $\mathfrak{I}$ interacts with a sequential-fast environment that may submit external actions sequentially before the system has reached the next stable state. In this case, a sequence of external actions may create races even if each individual action is race-free in a corresponding stable state. An external transition can be enabled in some transient global state where an internal transition is also enabled. This may create a race.

Let $\sigma$ be a sequence of $k$ external actions, $\sigma=a_{1} \ldots a_{k} \in A_{\text {ext }}{ }^{*}$. Let $\operatorname{Tr}_{S_{t_{\mathrm{g}}}}(s)$ denote the set of traces of stable state $s$ of stable composite machine $S t_{\Im}$. 
Given stable global state $s$ and a sequence $\sigma$ of $k$ external actions, $\sigma=a_{1} \ldots a_{k}$ $\in A_{\text {ext }}{ }^{*}$, such that $\sigma \in \operatorname{Tr}_{S_{t_{3}}}(s)$, we define (s after-ext $\sigma$ ) to be the set of all possible stable global states that can be reached in $C_{\mathfrak{I}}$ when the external trace $\sigma$ is executed from $s$ within a sequential-fast environment, i.e., (s after-ext $\sigma$ ) $=\left\{s^{\prime} \mid \exists \beta_{1}, \ldots, \beta_{k} \in A_{\text {int }}^{*}: s^{\prime} \in\left(s\right.\right.$ after $\left.a_{1} \beta_{1} \ldots a_{k} \beta_{k}\right) \wedge$ enabled $\left.\left(s^{\prime}\right) \subseteq A_{\text {ext }}\right\}$.

Definition 9. Given stable state $s$ and external trace $\sigma \in \operatorname{Tr}_{s t_{\mathfrak{J}}}(s)$,

- the trace $\sigma$ creates a race in $s$ if $\mid($ s after-ext $\sigma) \mid>1$;

- $\sigma$ is race-free in $s$ if $\mid(s$ after-ext $\sigma) \mid=1$.

The system $\mathfrak{I}$ is said to be race-free within a sequential-fast environment if in each stable state $s$ every $\sigma \in \operatorname{Tr}_{S_{t_{3}}}(s)$ is race-free.

If an external trace is race-free in some state, then it can be executed at this stable state in a speed-independent manner (see Section 4.2). The final stable state is always the same regardless of the speed at which the actions are consecutively executed. Similar to a sequential-slow environment (cf. Proposition 3), there exists a simple sufficient condition for the absence of races in the case of a sequential-fast environment.

Proposition 4. Concurrent system $\mathfrak{I}$ is race-free within a sequential-fast environment if in each local state all outgoing transitions are external or there is a single internal outgoing transition.

The stable composite machine does not represent m-race conditions, and the presence of races within a sequential-fast environment can be verified by constructing a part of the composite machine as follows.

Proposition 5. Given a stable state $s=\left(s_{1}, \ldots, s_{n}\right)$, let $\sigma=a_{1} \ldots a_{k}$ be an external trace $\sigma \in \operatorname{Tr}_{S t_{3}}(s)$ and $E(\sigma)$ denote the linear LTS such that it has $k+1$ states and a single completed trace $\sigma$. The trace $\sigma$ is race-free in $s$ iff the system $E(\sigma) \|\left(M_{1}\left(s_{1}\right)\|\ldots\| M_{n}\left(s_{n}\right)\right)$ has a single deadlock state.

Here, the notation $M(s)$ refers to LTS $M$ initialized in state $s$. As this statement indicates, the problem of race detection is computationally as difficult as the classical reachability problem.

\section{EXAMPLE}

Consider the system $E(b b)\|A(1)\| B(1) \| C(0)$, i.e. execution of the external trace $b b$ in the stable global state 110 (Figure 5). In the stable composite machine, i.e., under the sequential-slow environment assumption, the trace takes the system into the unique stable state 000 . Not so within a sequential- 
fast environment since three possible final states can be reached. Hence the trace $b b$ creates a race in state 110 under the sequential-fast environment assumption. Consider another external trace $a b$ in state 000 , the system $E(a b) \|$ $A(0)\|B(0)\| C(0)$ has only one deadlock state in stable state 001 (cf. Figure 3 ), therefore $a b$ is race-free in state 000 .

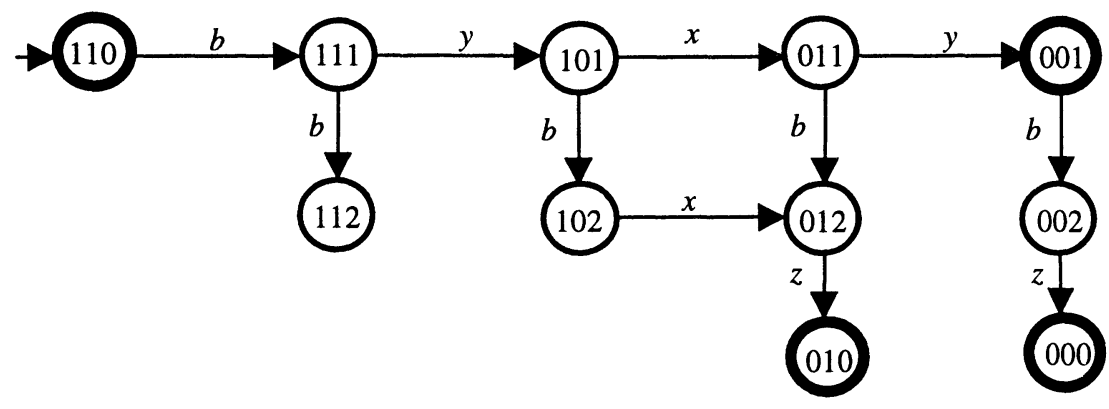

Figure 5. A race in state 110 for the sequence $b b$.

By definition, an m-race condition arises when an external and an internal action caused by a previous external action can be both executed in some (transient) global state. It is intuitively clear that such a condition does not lead to a race if external actions excite internal actions in different components. This observation leads us to the following sufficient conditions for the absence of races in external action sequences executed within a sequentialfast environment.

Let (s act-ext $a$ ) denote the set of all components that execute internal transitions in response to an external action $a$ applied at stable state $s$, i.e., ( $s$ act-ext $a)=\left\{i \in \operatorname{id}(b) \mid b \in A_{\text {int }} \wedge \exists \beta \in A_{\text {int }}{ }^{*}: a \beta b \in \operatorname{Tr}_{s c_{\mathfrak{I}}}(s)\right\}$, where $\operatorname{Tr}_{s_{c_{\mathfrak{I}}}}(s)$ is the set of traces of a slow-composite machine $S c_{\mathfrak{g}}$.

Proposition 6. Given a stable state $s$ and external trace $\sigma=a_{1} \ldots a_{k} \in A_{\text {ext }}{ }^{*}$, let $s_{1} \ldots s_{k} s_{k+1}$ be the corresponding sequence of traversed stable states, where $s=$ $s_{1}$. The trace $\sigma$ is race-free in $s$ if for all $i, 1 \leq i \leq k$, the action $a_{i}$ is race-free in state $s_{i}$ and $\left(s_{i}\right.$ act-ext $\left.a_{i}\right) \cap \cup_{j<i}\left(s_{j}\right.$ act-ext $\left.a_{j}\right)=\varnothing$.

\subsection{Concurrent Environment}

We now consider a concurrent environment that can simultaneously execute several external actions of different components, however only one action at a time for a particular component. Assuming that the concurrent system comprises $n$ components, the concurrent environment can simultaneously execute at most $n$ external actions with the system as a single 
concurrent action. To be consistent with the interleaving semantics, we represent a concurrent action with $k$ elementary actions, $k \leq n$, by an LTS with $2^{k}$ states (a hypercube), each completed trace of it is one possible ordering (permutation) of length $k$ of all the constituent external actions.

To constitute a concurrent action, it is required that two actions $a, b \in$ $A_{\text {ext }}$ have disjoint occurrence sets, i.e., $\operatorname{id}(a) \cap i d(b)=\varnothing$ (the actions are independent). This is a syntactic sufficient condition for concurrent transitions used in [5] that can be applied also in our context: two independent external actions $a$ and $b$ can be executed as a concurrent action if in a given stable state neither $a$ nor $b$ enable subsequent internal actions. However if the concurrent system has internal actions, it is not guaranteed that two independent external actions executed concurrently will result in the same final stable state. Therefore, we need a stronger requirement to define concurrent actions.

Definition 10. Given a deterministic stable composite machine $S t_{\Im}$, two external actions $a, b \in A_{\text {ext }}$ are potentially concurrent in stable state $s \in S_{S t}$ if : 1. $a, b \in \operatorname{enabled}(s), b \in \operatorname{enabled}\left(s\right.$ after $\left._{S t} a\right), a \in \operatorname{enabled}\left(s\right.$ after $\left._{S t} b\right)$ and 2. $s=a b \Rightarrow_{s t} s^{\prime}, s=b a \Rightarrow_{s t} s^{\prime \prime}, s^{\prime}=s^{\prime \prime}$.

The above two conditions, (1) enabledness and (2) commutativity, are similar to the conditions of independence relation given in [7], [5]. The difference is that we formulate them in terms of a stable composite machine and not, as it is usually the case in verification, of a composite machine. Moreover, in our context, to be truly concurrent, potentially concurrent actions should not create races. Given a subset of potentially concurrent external actions $P$, we use $[P]$ to denote the set of all possible linearization traces of the constituent actions, i.e. all of its permutations.

Definition 11. Given stable state $s$ and a set $P$ of external actions that are in pairs potentially concurrent, $P \subseteq \operatorname{enabled}(s)$,

- the set $P$ creates a race in $s$ if $\mid \cup_{\sigma \in[P]}(s$ after-ext $\sigma) \mid>1$,

- $P$ is race-free in $s$ if $\| \cup_{\sigma \in[P]}(s$ after-ext $\sigma) \mid=1$.

If set $P$ is race-free, then we also say that $P$ is a concurrent action in state $s$.

Proposition 7. Given a stable state $s$ and a set $P \subseteq \operatorname{enabled}(s)$ containing in pairs potentially concurrent external actions, the set $P$ is a concurrent action in $s$ iff all possible linearizations $\sigma \in[P]$ are race-free in $s$. 
Proof. If the set $P$ is a concurrent action in $s$ then the union $\cup_{\sigma \in[P]}(s$ afterext $\sigma$ ) is a singleton. The latter implies that the set ( $s$ after-ext $\sigma$ ) is also a singleton for all $\sigma \in[P]$. We conclude that each $\sigma \in[P]$ is race-free in $s$.

To demonstrate the other part of the statement, we assume that there are two potentially concurrent external actions $a, b \in P$ such that $a b$ are $b a$ are race-free in $s$, but the set $\langle a, b\rangle$ (and thus the set $P$ ) creates a race in $s$. We have $\mid(s$ after-ext $a b) \cup(s$ after-ext $b a) \mid>1$, at the same time $a$ and $b$ are potentially concurrent in state $s$. The commutativity property of actions $a$ and $b$ of a deterministic system implies that $s=a b \Rightarrow_{s t} s^{\prime}, s=b a \Rightarrow_{s t} s^{\prime \prime}, s^{\prime}=s^{\prime \prime}$. Then $s^{\prime}=((s$ after-ext $a)$ after-ext $b)=((s$ after-ext $b)$ after-ext $a)=s^{\prime \prime}$ holds. Taking into account that $\mid(s$ after-ext $a b) \cup(s$ after-ext $b a) \mid>1$, we conclude that either $\mid(s$ after-ext $a b) \mid>1$ or $\mid(s$ after-ext $b a) \mid>1$. That means, trace $a b$ or $b a$ should create a race in $s$, which contradicts our assumption that $a b$ and $b a$ are race-free in $s$.

\section{EXAMPLE}

In our working example (cf. Figure 4), we have two external actions $a$ and $b$. They cannot be potentially concurrent in stable state 110 , since $a \notin$ enabled (110), neither can they in stable state 001 , for $001=a b \Rightarrow_{s t} 000$ but $001=b a \Rightarrow_{s t} 010$. On the other hand, it can be verified that they constitute a concurrent action $\langle a, b\rangle$ in the states 000 and 010 of the stable composite machine.

A sufficient condition can be formulated for a pair of potentially concurrent external actions to form a concurrent action taking into account the components that participate in the execution of each action.

Proposition 8. Given a stable state $s$ and external actions $a, b \in \operatorname{enabled}(s)$, $<a, b\rangle$ is a concurrent action in state $s$ if $($ s act-ext $a) \cap($ s act-ext $b)=\varnothing$.

E-race conditions can be verified by a direct construction of a part of the composite machine instead of verifying each linearization as suggested by Proposition 7. This fact is expressed by the following proposition.

Proposition 9. Given a stable state $s=\left(s_{1}, \ldots, s_{n}\right)$, let $P$ be a set of external actions that are in pairs potentially concurrent in $s$ and $E(P)$ denote an LTS such that it contains $2^{|P|}$ states and the set of all of its completed traces is $[P]$. The set $P$ is a concurrent action in $s$ iff the system $E(P) \|\left(M_{1}\left(s_{1}\right)\|\ldots\|\right.$ $\left.M_{n}\left(s_{n}\right)\right)$ has a single deadlock state. 
Up to here we have established all necessary definitions and propositions needed to perform a race analysis for a concurrent system of communicating LTSs. The next section demonstrates how this knowledge helps deal with races during testing of such systems.

\section{TEST EXECUTION AVOIDING ACTION RACES}

\subsection{Test Architecture and Testing Goal}

The test architecture to test concurrent system $\mathfrak{I}$ consists of the parallel composition of the system under test (SUT), the implementation of $\mathfrak{I}$, and a tester modeled in terms of one or several communicating LTSs. The tester is able to observe and control all actions of the SUT, which it has access to (synchronous communication). In opposition to [10] we ease the assumption that the tester participates in all internal and external actions of the SUT. Instead we assume that the SUT exposes only its external actions to the tester. Consequently, race conditions between external test actions and/or internal actions of the SUT might occur during a test run.

Testing implementations of a concurrent system specification with respect to races, we may intend to verify whether or not a test case of an external action trace that is race-free in the specification is also race-free in the implementation. Such a testing goal is interesting since opposed to a typical testing assumption, an implementation might be in this case "more nondeterministic" than its specification. Since races cause nondeterministic behavior, their guaranteed detection requires that a certain fairness assumption (often called complete testing assumption) is satisfied for the implementation. To obtain confidence that the SUT contains no additional races than given in its specification, we need to rerun the same test several times and to identify the global state reached of the SUT after each test run. These tests must be deterministic, i.e., they do not contain any known specification races, to clearly indicate deviating behavior and thus faults of the SUT. Besides the detection of further races in an implementation, deterministic, racefree tests greatly reduce test efforts since the test purpose of a test case can be definitely reached for a correct SUT, which is hardly possible for nondeterministic tests.

In this section, we consider how testers should be devised that execute test cases at the highest possible speed without triggering specification races in order to detect additional races in the SUT and possibly other faulty behavior. 


\subsection{Steady Testing}

Given a test case as an external action sequence $\omega$ over $A_{\text {ext }}$ for concurrent system $\mathfrak{I}$, the execution of the test case on the SUT of $\mathfrak{I}$ is usually modeled as a parallel composition with the II operator of the tester $T(\omega)$ implementing $\omega$ as a linear LTS and the SUT itself.

Deterministic test cases exists only when i-race conditions in the specification do not lead to races, i.e., the stable composite machine of the specification is deterministic (see Section 3.2). We assume that it is always the case. To avoid further specification races within a sequential-slow environment, the tester must guarantee that the SUT always returns into a stable state before the next external action of the test case is applied to the SUT. This behavior is achieved if the $\rfloor\lfloor$ operator is applied between tester and SUT instead of $I I$. We refer to this mode of testing as steady testing.

To implement the $\rfloor\lfloor$ operator, a tester implementing a test case has to use delays, denoted as $\Delta$, to insure that the SUT reaches a stable state in response to a previous external test action ${ }^{1}$. Consider our example system and assume the test designer derives the test case $\omega=a b a b a a b b b b$ (a transition tour through the stable composite machine from Figure 4), then the test case for system $\mathfrak{I}$ executed under the slow environment assumption is $\omega_{\text {steady }}=a$ $\Delta b \Delta a \Delta b \Delta a \Delta a \Delta b \Delta b \Delta b \Delta b$.

\subsection{Sequential-Fast Testing}

To increase the execution speed of tests, one has to use a tester that behaves as a sequential-fast environment to the SUT provided that specification races do not occur. Formally speaking, we have to derive maximum race-free test steps (nonempty sub-sequences) $\omega_{1}, \omega_{2}, \ldots$ from the original test case $\omega$, such that $\omega=\omega_{1} \omega_{2} \ldots$. Then delays have to be inserted only after each test step, $\omega_{\text {seq }}=\omega_{1} \Delta \omega_{2} \Delta \ldots$. The longer these test steps are the fewer delays have to be inserted to avoid specification races. The resulting test can be executed faster in most cases than the previous secure test in steady testing that requires inserting delays after each single test action.

Thus, given test case $\omega$, we have to determine a minimum number of delays or, in other words, a minimum partitioning of $\omega$ in order to execute this

1 Note that delays cannot be expressed in our LTS model quantitatively since it does not support time. Therefore the value of a delay must be determined by other means. The delay depends mainly on the execution time of messages exchanged between two participating components and on the length of the execution sequence of internal actions. The test engineer needs to adjust this time for any specific system. 
sequence in the fastest sequential way. More formally, find a partitioning $\omega_{1}$, $\ldots, \omega_{r}$ of $\omega$ such that:

1. $\omega_{1} \ldots \omega_{r}=\omega$

2. $\omega_{1}$ is race-free in the initial stable state $s_{03}$ and $\omega_{i}$ is race-free in stable state $\left(s_{0 \Im}\right.$ after-ext $\left.\left(\omega_{1} \ldots \omega_{i-1}\right)\right)$ for all $i>1$; and

3. if there exist another partitioning $\omega_{1}^{\prime}, \ldots, \omega_{k}^{\prime}$ such that (1) and (2) are satisfied, then $k \geq r$.

\section{Algorithm 1.}

1. The initial stable state is $s_{03}$.

2. Find a maximum race-free prefix of $\omega$ in the given initial stable state by analyzing the sequence of the first two consecutive actions to be racefree using Proposition 5 and then consecutively adding actions one by one until the sequence is no longer race-free.

3. Remove the obtained prefix from $\omega$ and do Step 1 starting from the stable state reached by the previous prefix until $\omega$ is finished.

Proposition 10. Algorithm 1 yields a minimum race-free partitioning of $\omega$.

Proof. Let us assume that the sequence $\omega$ is partitioned into $n$ race-free nonempty sequences, $\omega=\omega_{1} \omega_{2} \ldots \omega_{n}$. Suppose there exists a partitioning of $n-1$ race-free sequences only, $\omega=v_{1} v_{2} \ldots v_{n-1}$. In this case, there should exist two subsequences $\omega_{i}$ and $v_{j}$ such that $\alpha \omega_{i} \beta=v_{j}$, where $\beta$ is a non-empty sequence, otherwise the second partitioning cannot have fewer sequences. Consider the case when $\alpha$ is an empty sequence. Then $\omega_{i}$ cannot be extended by a non-empty sequence $\beta$ to obtain a race-free sequence $\omega_{i} \beta$, for Algorithm 1 delivers the longest race-free sequence in stable state $\left(s_{0 \Im}\right.$ after-ext $\omega_{1} \omega_{2}$ $\left.\ldots \omega_{i-1}\right)$. If $\alpha$ is a non-empty sequence, then $v_{j}=\alpha \omega_{i} \beta$ must be race-free in stable state $\left(s_{0 I}\right.$ after-ext $\left.v_{1} v_{2} \ldots v_{j-1}\right)$. This implies that $\omega_{i} \beta$ is race-free in stable state $\left(s_{0 \Im}\right.$ after-ext $\left.v_{1} v_{2} \ldots v_{j-1} \alpha\right)$. Furthermore, we have ( $s_{03}$ after-ext $\left.v_{1} v_{2} \ldots v_{j-1} \alpha\right)=\left(s_{03}\right.$ after-ext $\left.\omega_{1} \omega_{2} \ldots \omega_{i-1}\right)$. This means that $\omega_{i} \beta$ is race-free in stable state $\left(s_{03}\right.$ after-ext $\left.\omega_{1} \omega_{2} \ldots \omega_{i-1}\right)$, which is impossible since $\omega_{i}$ is the longest race-free sequence in stable state $\left(s_{03}\right.$ after-ext $\left.\omega_{1} \omega_{2} \ldots \omega_{i-1}\right)$.

Once it is proven that a subsequence (a test step during testing) does not cause specification races, the execution time of the whole test case can be immediately reduced by avoiding all the delays between the consecutive actions within the subsequence.

A tester implementation of the partitioned test case $\omega_{\text {seq }}=\omega_{1} \Delta \omega_{2} \Delta \ldots$ is a deterministic global tester that has control to all external actions $A_{\text {ext }}$ of SUT. The global tester executes a test action one after another and performs 
additional delays as indicated in $\omega_{\text {seq }}$ to ensure that the SUT reaches a stable state from where the test is resumed. Consider our example system again, it can be analyzed that the same test case $\omega=a b a b a a b b b b$ can be partitioned into four test steps that are race-free in a sequential-fast environment. Therefore, it is sufficient to execute the test case $\omega_{\text {seq }}=a b a \Delta b a \Delta a b \Delta b b b$ to guarantee a deterministic sequential-fast test run without specification races. Instead of nine delays in $\omega_{\text {steady }}$ only three are needed in $\omega_{\text {seq. }}$.

\subsection{Concurrent Testing}

The results obtained in the previous section rely on sequential testers only. Further timesaving in the execution of tests is possible if concurrency is introduced in the tester. We discuss the interplay of concurrency and specification races first for the abstract notion of a global concurrent tester and next for the model of a distributed concurrent tester that can be the basis for practical tester implementations.

To start with, we consider a global concurrent tester that can simultaneously execute several external actions with different components, however only one action at a time for a particular component. That means, such a tester can execute several external actions with the system as a single concurrent action as discussed in Section 3.4.

We assume that a test case is partitioned into several consecutive test steps using the analysis method presented in Section 4.2. Additionally, each of the test steps may contain concurrent actions according to Section 3.4. If concurrent actions exist in a test step, we refer to it as a concurrent test step. Delays are inserted only between the test steps of the test case. A delay inserted between two test steps indicates that the tester must slow down to allow the concurrent system to stabilize in the next stable state in order to avoid specification races.

A concurrent test step for concurrent system $\mathfrak{I}$ is an acyclic LTS $T$ with the initial state $t_{0 T}$ and a single sink state. Its set of actions coincides with $A_{\text {ext }}$ of $\mathfrak{I}$. If the test step is a single concurrent action, the LTS $T$ is a hypercube (see Section 3.4). E-race conditions occur if there is a state in $T$ with more than one action enabled, i.e., if the test step contains concurrent actions. To verify race conditions between tester and specification, a part of their composite machine has to be constructed, similar to Proposition 9.

Proposition 11. Given a concurrent test step $T$ for $\mathfrak{I}$ in stable state $s$, test step $T$ is race-free in $s$ iff the system $T\left(t_{0}\right) \|\left(M_{1}\left(s_{1}\right)\|\ldots\| M_{n}\left(s_{n}\right)\right)$ has a single deadlock state. 
When specification races are detected for a given concurrent test step, it has to be modified to ensure a deterministic test run. The composition $T\left(t_{0}\right) \|$ $\left(M_{1}\left(s_{1}\right)\|\ldots\| M_{n}\left(s_{n}\right)\right)$ helps identify traces of $T$ that have to be serialized to avoid specification races. In the extreme situation, a concurrent test step might be transformed into a race-free test sequence.

\section{EXAMPLE}

Taking the initial partitioning of the (sequential) test case for our example system from Section $4.2 \omega_{\text {seq }}=a b a \Delta b a \Delta a b \Delta b b b$, an analysis for concurrent actions reveals the concurrent test case $\omega_{\text {conc }}=\langle a, b\rangle a \Delta b a \Delta<a$, $b>\Delta b b b .^{2}$ The savings to use a concurrent tester instead a sequential-fast one are two concurrent actions, i.e., the length of the test case (including delays) could be reduced from 19 test actions for a sequential-slow tester to 13 test actions for a sequential-fast tester to 11 test actions for the concurrent tester.

The global concurrent tester can be refined such that concurrent actions are executed truly concurrent instead of executing them in an arbitrary interleaving order. To obtain true concurrency, the tester must be distributed among several concurrent tester components. We assume that such a distributed tester comprises $k$ tester components, where $k$ is the number of external ports of system $\mathfrak{I}$. Each tester components controls and observes a subset of external actions from $A_{\text {ext }}$. That means, a distributed tester is another concurrent system $T$ with the same alphabet of external action $A_{\text {ext }}$ as concurrent system $\mathfrak{I}$.

To support collaboration among the distributed tester components, a synchronization mechanism must be implemented. If broadcast communication is available, a single synchronization action is used as an internal action of the distributed tester $\boldsymbol{T}$. In case of multicast, the alphabet of internal actions of the system $\boldsymbol{T}$ consists of several synchronization actions for each necessary subset of tester components that needs to be synchronized. Each tester component is modeled as an LTS with the unobservable action $\tau$ that represents a delay needed to avoid races in a test run. The global behavior of the distributed tester is given by the parallel composition of its tester components, $T=T_{1}\|\ldots\| T_{k}$.

Once a distributed tester is constructed for a given concurrent test case, it can be verified whether it contains race conditions using the results presented

2 Note that the partitioning $b a$ after the first delay does not constitute a concurrent action, although $a$ and $b$ are independent. However, the two possible linearizations $b a$ and $a b$ starting from stable state 001 result in the two different stable states 010 and 000 , respectively, which can be verified using Figure 4 (cf. Definition 10). 
in previous sections. A distributed tester is usually required to behave deterministically (unless the test designer intentionally opts for nondeterministic testing). This means that its internal synchronization actions should not create races. A stable composite machine of the distributed tester has to be deterministic.

The approach in [6] requires, similar to our work, that the parallel composition of tester components (after hiding) is a given test case. It neglects however the possibility of races inside the tester and between tester and SUT. Races must be completely avoided to ensure a deterministic test run. One can say that a deterministic concurrent distributed tester exemplifies the design of a concurrent system without races.

\section{CONCLUSIONS}

The paper introduced the issue of action races as an inherent property of concurrent systems. Such races occur when internal and external actions of the system are enabled in certain global states that result in different end states. Three different assumptions on the environment of a concurrent system and their influence on race conditions were discussed. A very stringent assumption is the sequential-slow environment where only race conditions between internal actions occur. However the execution time of the concurrent system is artificially inflated.

A more realistic environment is a sequential-fast environment that can perform several external actions sequentially before the system reaches the next stable state. Here mixed race conditions between internal and external actions may occur, their absence has to be verified in the specification in order to guarantee a deterministic run of the concurrent system. Finally a concurrent environment was introduced that represents what seems to be the most universal environment. In addition to mixed race conditions, race conditions between external actions may occur due to the concurrent execution of these actions.

Moreover, the paper presented necessary and sufficient conditions to avoid races in a concurrent system. Still, races may be not completely avoided in the design of a concurrent system. In spite of this, races have to be identified to take preventive measures in the implementation of the system. The issue of races is of interest too when it comes to testing concurrent systems. We demonstrated how the analysis of races helps design testers that execute test actions without races at a highest possible speed.

The paper presented only initial results in the field of race analysis based on specifications. More work needs to be done. More subtle conditions 
should be established to detect race conditions in a concurrent system. It is also interesting to study the interplay of verification techniques based on partial orders and race analysis techniques proposed in this paper. Another interesting topic is to study the test generation process that avoids specification races. Last but not least, race analysis must be discussed in the context of communicating I/O-LTSs in an asynchronously communicating environment to address more realistic systems.

\section{ACKNOWLEDGEMENT}

The first author acknowledges support of NSERC grant OGP0194381.

\section{References}

[1] R. Alur, G. Holzmann, D. Peled: An Analyzer for Message Sequence Charts; 2nd Int'1 Workshop TACAS'96, 1996; pp. 35-48.

[2] K. Audenaert: Maintaining Concurrency Information for On-the-fly Data Race Detection; Parallel Computing 1997; Bonn, Germany, September 1997; pp. 19-22.

[3] J. A. Brzozowski, C.-J. Seger: Asynchronous Circuits; Springer-Verlag, 1994; 404 p.

[4] A. Bechini, K. C. Tai: Timestamps for Programs Using Messages and Shared Variables; 18th Inter'l Conference on Distributed Computing Systems; Amsterdam, The Netherlands, May 1998; pp. 266-273.

[5] P. Godefroid: Partial-order methods for the verification of concurrent systems; LNCS 1032; Springer, 1996.

[6] C. Jard, T. Jeron, H. Kahlouche, C. Viho: Towards automatic distribution of testers for distributed conformance testing; FORTE/PSTV'98; Paris, France, 1998.

[7] S. Katz, D. Peled: Defining conditional independence using collapses. Theoretical Computer Science, vol. 101, 1992; pp. 337-359.

[8] G. Luo, G. v. Bochmann, A. Das, Ch. Wu: Failure-equivalent transformation of transition systems to avoid internal actions; Information Processing Letters, vol. 44 (1992), pp. 333-343.

[9] R. H. Netzer, B. P. Miller: Optimal Tracing and Replay for Debugging MessagePassing Parallel Programs; Conference on Supercomputing 1992; pp. 502-511.

[10] A. Petrenko, A. Ulrich, V. Chapenko: Using partial-orders for detecting faults in concurrent systems; IWTCS'98, Tomsk, Russia, 1998; pp. 175-190.

[11] K. C. Tai: Race analysis of traces of asynchronous message-passing programs. 17th Int'l Conference on Distributed Computing Systems (ICDCS'97); Baltimore, MD, USA, 1997; pp. 261-268. 